\title{
Effect of an innovative community-based care model, the Monarch Centre, on postpartum length of stay: an interrupted time-series study
}

\author{
Ghislain Hardy MD, Jo Ann Colas MSc, Deborah Weiss PhD, David Millar MD, Alan Forster MD, \\ Mark Walker MD, Daniel J. Corsi PhD
}

See related commentary at www.cmaj.ca/lookup/doi/10.1503/cmaj.180836

Abstract

Background: Reduction in postpartum length of stay has been advocated within a context of reducing health care system costs and maintaining quality of care. We assessed trends in postpartum length of stay for vaginal and cesarean deliveries at an academic hospital, The Ottawa Hospital, before and after the implementation in 2014 of a novel community-based postpartum outpatient clinic, the Monarch Centre.

Methods: The Monarch Centre model of postpartum care consists of prebooked appointments at the postpartum clinic, scheduled within 48 hours of hospital discharge. Clients receive maternal assessment including mood screening and care, neonatal care, laboratory testing including infant total serum bilirubin level, and breast-feeding assessment and support. Family physicians, lactation consultants and registered nurses are available for consultation at the appointment, and there is coordination with institutional care, community partners and primary care providers. We used interrupted time-series regression models to assess trends in postpartum length of stay at The Ottawa Hospital between January 2012 and December 2016.

Results: There were 16023 deliveries with 16515 babies born over the study period. The mean postpartum length of stay was 46 hours ( $66 \mathrm{~h}$ for cesarean deliveries and $37 \mathrm{~h}$ for vaginal deliveries). Eighteen months after implementation of the centre, the average length of stay following a cesarean birth had decreased by 20 hours, a relative reduction of $27 \%$ (95\% confidence interval [Cl] 9.5 to 30.4$)$; for vaginal deliveries, length of stay was reduced by 6 hours, a relative reduction of $18 \%(95 \% \mathrm{Cl} 5.2$ to 31.1$)$, and by 12 hours among typical cases (relative reduction $28 \%, 95 \% \mathrm{Cl} 19.2$ to 36.6 ). There was a decrease in the proportion of women with a length of stay exceeding 48 hours after centre implementation. An increase in the rate of 30 -day readmission from $1.1 \%$ to $1.9 \%$ was observed among babies.

Interpretation: A strong association was found between implementation of a community-based multidisciplinary postpartum clinic and declines in postpartum length of stay. This alternative model of postpartum care is safe, has the potential for reducing provider costs and should be considered for further implementation at provincial and national levels.

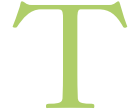

he Canadian health care system faces serious economic challenges to meet the demands of an aging population. Health care services are the largest component in every provincial and territorial budget in Canada. ${ }^{1}$ Hospital and inpatient care represent the largest proportion of health care expenses, and giving birth is the most frequent reason for hospital admission. ${ }^{2}$ In 2015/16, there were 368676 inpatient hospital admissions attributed to childbirth, with an average length of stay of 2.3 days. $^{2}$ Therefore, new models of obstetrical patient care that promote shorter length of stay, high-quality care and patient satisfaction, and decrease postpartum care costs are being considered. ${ }^{3}$ Postpartum care following hospital discharge is of utmost importance for women and their newborns as it helps lead to a smooth transition from hospital care back to home life and contributes to improving health outcomes, although the optimal model has yet to be established., ${ }^{4,5}$ For many women in Canada, available outpatient postpartum support appears to be limited, which can present numerous challenges. For instance, new mothers may experience

Competing interests: None declared.

This article has been peer reviewed.

Correspondence to: Daniel Corsi, dcorsi@ohri.ca

CMAJ Open 2018. DOI:10.9778/cmajo.20180033 
difficulties in establishing breast-feeding, which can lead to neonatal jaundice, poor weight gain, dehydration resulting in increased visits to the emergency department, longer hospital stays and breast-feeding cessation. ${ }^{6-10}$

Early discharge in combination with an individualized follow-up program has shown safe outcomes for both the neonate and the woman. ${ }^{11,12}$ Certain programs that often involve frequent postpartum home visits by nurses or other health care professionals have been shown to improve family outcomes and patient satisfaction, and decrease infant emergency care episodes and hospital admission. However, these types of care models are not always accessible in every community, require substantial human resources and are typically very costly. ${ }^{13}$ An innovative community-based postpartum clinical care service, the Monarch Centre, was introduced and evaluated in our obstetrical centre in Ottawa in 2014. In this program, women and their babies receive multidisciplinary postpartum care in an outpatient clinic during the first month following hospital discharge after delivery. In a randomized controlled trial evaluating mother-baby dyads receiving this model of care, we showed a high level of satisfaction among women and no difference in rates of emergency department visits compared to a control group receiving standard therapy. ${ }^{14}$ The objective of the present study was to further evaluate the effect of referral to the Monarch Centre on postpartum length of stay at The Ottawa Hospital. We present trends in postpartum length of stay at the Civic Campus of The Ottawa Hospital before and following implementation of the centre.

\section{Methods}

\section{Data}

Data originated with The Ottawa Hospital Data Warehouse, a relational database that contains administrative and clinical data for all patients at The Ottawa Hospital and has been previously used for research. ${ }^{15}$ For this study, data from the The Ottawa Hospital patient registration system, the Canadian Institute for Health Information's Discharge Abstract Database and National Ambulatory Care Reporting System Metadata were extracted from the The Ottawa Hospital Data Warehouse for the period 2012-2016. The patient registration system contains patient demographic information along with dates and times of admissions and discharges.

\section{Intervention and implementation period}

The Monarch Centre model of postpartum care consists of prebooked appointments at the postpartum clinic, scheduled within 48 hours of hospital discharge, with additional follow-up visits for missed appointments. The clinic visits involve maternal assessment including mood screening and care (e.g., wound care, prescriptions), neonatal care (e.g., weight gain assessment, jaundice screening with a transcutaneous bilirubinometer), laboratory tests including infant total serum bilirubin level, and breast-feeding assessment and support. Family physicians, lactation consultants and registered nurses are also available for consultations at the appointment, and there is coordination with institutional care (e.g., hospital postpartum wards and neo- natal intensive care unit), community partners (e.g., public health) and primary care providers (family physicians and pediatricians) to assist families in establishing postdischarge care. ${ }^{14}$

This study covers the 5-year period Jan. 1, 2012, to Dec. 31, 2016. The Monarch Centre was launched at The Ottawa Hospital in January 2014 as a 6-month trial program and was fully implemented over the following year. Therefore, to properly evaluate the impact of the centre on postpartum length of stay, we treated the period Jan. 1, 2014, to July 31, 2015 as the implementation period for the analysis. The preimplementation period was Jan. 1, 2012, to Dec. 31, 2013, and the postimplementation follow-up period was Aug. 1, 2015 to Dec. 31, 2016.

\section{Outcome}

The primary outcome was postpartum length of stay. We aggregated record-level data to 4-week intervals (which accounts for differing number of days in months) for timeseries analyses (over a total study duration of $60 \mathrm{mo}$ ). For secondary outcomes, we examined the proportion of women with a length of stay longer than 48 hours and the rate of 30-day unplanned readmission for women and babies.

\section{Statistical analysis}

We carried out descriptive analyses to look at the distribution of demographic factors, type of delivery, procedures and differences in mean length of stay across study periods. The main analyses used an interrupted time-series segmented regression design. This is a robust, quasi-experimental approach for evaluating the longitudinal effects of interventions. ${ }^{16}$ Furthermore, the use of segmented analysis of interrupted time-series data allows for the assessment of differences in trends in length of stay due to the Monarch Centre. ${ }^{17}$

We specified interrupted time-series models to estimate level and trend in postpartum length of stay in the preimplementation period, and changes in level and trend in the postimplementation period. The following model was specified:

$y=\beta_{0}+\beta_{1} \times$ time $+\beta_{2} \times$ implementation $+\beta_{3} \times$ time after $+e$

where $y$ is length of stay in hours, $\beta_{0}$ represents baseline average postpartum length of stay, $\beta_{1}$ is the trend in the average postpartum length of stay before the implementation period, $\beta_{2}$ estimates the change in the average postpartum length of stay immediately after the implementation period, $\beta_{3}$ estimates the change in the trend in the average postpartum length of stay after the implementation period compared to the trend before the implementation period, and $e$ represents the random error.

All analyses were stratified by cesarean and vaginal delivery. We considered all deliveries and a subset of "typical" deliveries, defined as cases in which the woman was admitted to the hospital through the birthing unit and then, after delivery, was transferred to the mother/baby unit, from which she was routinely discharged as an obstetrics patient. This excluded women admitted to other units (e.g., intensive care unit, postanesthesia care unit) during the hospital stay, admitted to 
the hospital through the emergency department, or transferred back and forth from the birthing unit and the mother/baby unit while waiting for the start of the delivery. In addition, we conducted interrupted time-series analyses of 2 control outcomes that were not targeted and were unlikely to be affected by referral to the Monarch Centre if the association with postpartum length of stay is causal but are also subject to the same confounding structure as the outcome of interest: gestational age at admission and length of stay following hysterectomy. The analysis stratified the hysterectomy cohort by 3 subgroups with varying length of stay: inpatients, same-day admissions and day surgeries. Finally, we used the interrupted time-series model to estimate the long-term effect of implementation of the Monarch Centre on postpartum length of stay.

\section{Ethics approval}

The study was approved by the Ottawa Health Science Network Research Ethics Board.

\section{Results}

There were 16023 deliveries with 16515 babies born between Jan. 1, 2012, and Dec. 31, 2016 (Table 1). The mean postpartum length of stay was 46 hours; it was longer after cesarean delivery than after vaginal delivery ( $66 \mathrm{~h} \mathrm{v.} 37 \mathrm{~h}$ ). Maternal characteristics including maternal age at admission and parity in the vaginal and cesarean delivery groups were similar across the 3 study periods.

In the vaginal delivery group, there was a significant reduction in mean length of stay, from 40.2 hours in the preintervention period to 30.2 hours in the postintervention period $(p<0.001)$ in descriptive analyses. There was also a significant reduction in length of stay in the cesarean delivery group, from 71.4 hours to 55.8 hours $(p<0.001)$. Before implementation of the Monarch Centre, 1356 women (30.2\%) with a vaginal delivery had a length of stay greater than 48 hours, compared to 354 women $(11.5 \%)$ after implementation $(p<$ $0.001)$. The corresponding values for women with a cesarean delivery were 1927 (90.4\%) and 668 (48.4\%) ( $p<0.001)$.

\section{Interrupted time-series analyses of change in postpartum length of stay}

Among all cases of vaginal delivery, postpartum length of stay decreased over the preimplementation period (Figure 1). Implementation of the Monarch Centre was associated with a reduction in length of stay of 3.6 hours, although this effect was not statistically significant $(p=0.2)$ (Table 2). The resulting postimplementation slope was a decrease of 10.9 minutes/ month in length of stay after the implementation period $(p=$ $0.05)$. Among typical cases of vaginal delivery, the magnitude of the implementation effect was larger (reduction of $5.7 \mathrm{~h}$, $p=0.02)$; the postimplementation slope was a reduction of 10.6 minutes/month $(p=0.04)$. Among all cesarean deliveries, implementation of the Monarch Centre was associated with a decrease of 7.2 hours in average length of stay, although this was not statistically significant $(p=0.2)$, and the combined postimplementation slope was a reduction of 18.6 minutes/ month $(p=0.08)$. For typical cesarean deliveries, implementation of the centre was associated with a reduction in the average length of stay of 9.5 hours $(p=0.001)$, and the average length of stay decreased by 16.5 minutes/month after the implementation period $(p=0.005)$.

\begin{tabular}{|c|c|c|c|c|c|c|c|c|}
\hline \multirow[b]{2}{*}{ Variable } & \multicolumn{4}{|c|}{ Vaginal delivery; no. (\%) of cases* } & \multicolumn{4}{|c|}{ Cesarean delivery; no. (\%) of cases ${ }^{*}$} \\
\hline & $\begin{array}{c}\text { Before } \\
\text { implementation } \\
n=4487\end{array}$ & $\begin{array}{l}\text { Implementation } \\
n=3306\end{array}$ & $\begin{array}{c}\text { After } \\
\text { implementation } \\
n=3067\end{array}$ & $\begin{array}{c}\text { Total } \\
n=10860\end{array}$ & $\begin{array}{c}\text { Before } \\
\text { implementation } \\
n=2131\end{array}$ & $\begin{array}{l}\text { Implementation } \\
\quad n=1651\end{array}$ & $\begin{array}{c}\text { After } \\
\text { implementation } \\
n=1381\end{array}$ & $\begin{array}{c}\text { Total } \\
n=5163\end{array}$ \\
\hline No. of babies & 4543 & 3354 & 3109 & 11006 & 2276 & 1748 & 1485 & 5509 \\
\hline $\begin{array}{l}\text { Length of stay, } h \text {, } \\
\text { mean } \pm S D\end{array}$ & $40.2 \pm 22.9$ & $37.6 \pm 23.1$ & $30.2 \pm 19.3$ & $36.6 \pm 22.4$ & $71.4 \pm 20.1$ & $66.2 \pm 25.5$ & $55.8 \pm 34.5$ & $65.6 \pm 27.1$ \\
\hline $\begin{array}{l}\text { Length of stay, } \mathrm{h} \text {, } \\
\text { median (IQR) }\end{array}$ & $40(27-52)$ & $35(25-49)$ & $27(23-36)$ & $34(25-48)$ & $70(63-77)$ & $67(51-74)$ & $48(43-63)$ & $66(49-74)$ \\
\hline Length of stay $>48 \mathrm{~h}$ & $1356(30.2)$ & $854(25.8)$ & $354(11.5)$ & $2564(23.6)$ & $1927(90.4)$ & $1336(80.9)$ & $660(47.8)$ & $3923(76.0)$ \\
\hline \multicolumn{9}{|l|}{$\begin{array}{l}\text { 30-day readmission } \\
\text { rate }\end{array}$} \\
\hline \multicolumn{9}{|l|}{ Women } \\
\hline Eligible cases & $4478(99.8)$ & $3300(99.8)$ & 3055 (99.6) & $10833(99.8)$ & $2122(99.6)$ & $1641(99.4)$ & $1367(99.0)$ & $5130(99.4)$ \\
\hline Readmitted & $43(1.0)$ & $48(1.4)$ & $33(1.1)$ & $124(1.1)$ & $41(1.9)$ & $28(1.7)$ & $33(2.4)$ & $102(2.0)$ \\
\hline \multicolumn{9}{|l|}{ Babies } \\
\hline Eligible cases & 4537 (99.9) & 3351 (99.9) & $3094(99.5)$ & $10982(99.8)$ & $2274(99.9)$ & $1747(99.9)$ & $1481(99.7)$ & $5502(99.9)$ \\
\hline Readmitted & $62(1.4)$ & $70(2.1)$ & $67(2.2)$ & 199 (1.8) & $13(0.6)$ & $18(1.0)$ & $22(1.5)$ & $53(1.0)$ \\
\hline
\end{tabular}



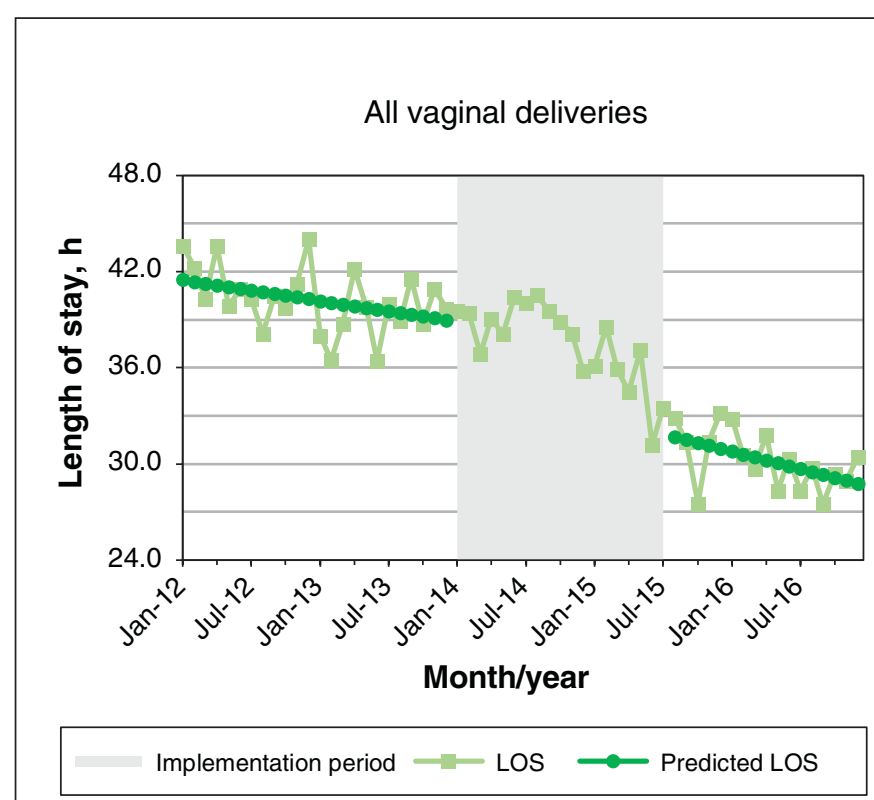

All cesarean deliveries



Typical vaginal deliveries

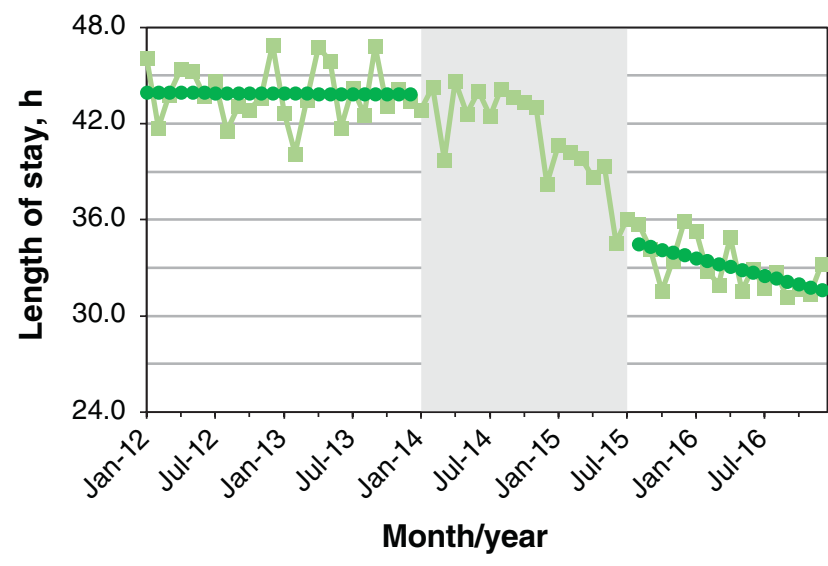

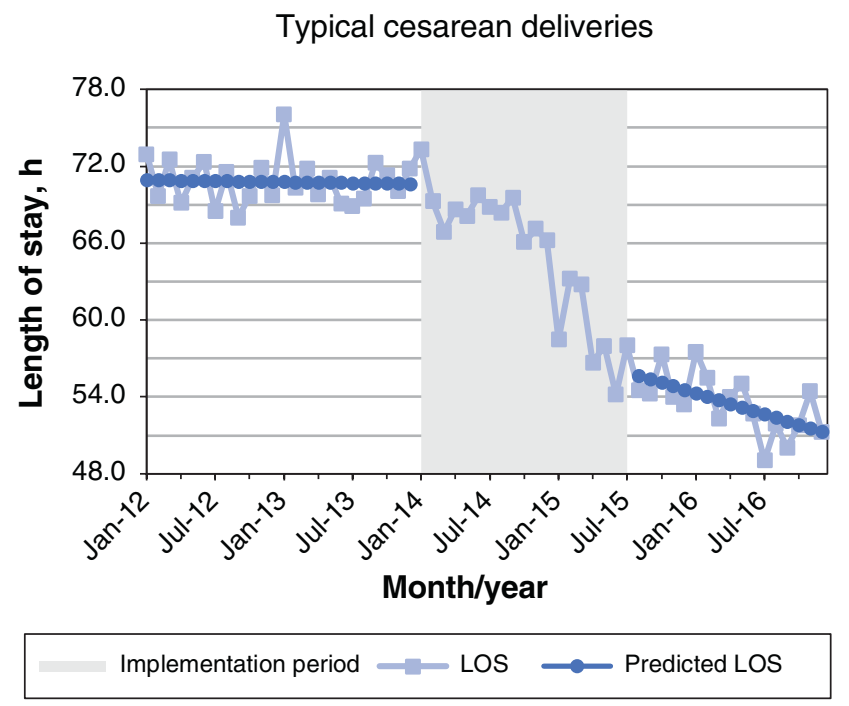

Figure 1: Actual and predicted average postpartum length of stay (LOS) by month for vaginal and cesarean deliveries at The Ottawa Hospital, January 2012 to December 2016. Typical deliveries defined as admissions to hospital through the birthing unit and direct transfer to mother/ baby unit before discharge; excludes women admitted to other units during the hospital stay and multiple transfers before delivery.

According to interrupted time-series models, at the start of the study period, $91.3 \%$ of women with typical cesarean deliveries had a postpartum length of stay greater than 48 hours, compared to $65.6 \%$ immediately after the implementation period (Figure 2), a relative decrease of $28.1 \%$ ( $p=$ $0.003)$. One-third $(33.5 \%)$ of women with typical vaginal deliveries had a length of stay greater than 48 hours at the start of the study period, compared to $23.1 \%$ immediately after the implementation period, a relative reduction of $31.0 \%$; however, the difference was not statistically significant $(p=0.06)$.

\section{Rates of 30-day readmission}

Overall rates of 30 -day readmission were $1.1 \%$ and $2.0 \%$ for women and $1.8 \%$ and $1.0 \%$ for babies in the vaginal and cesarean birth groups, respectively. Among women, 30-day readmission rates were stable across the study period in the vaginal $(p=0.1)$ and cesarean $(p=0.4)$ birth groups. Among babies, there was an increase in the rate of 30-day readmission between the preintervention period and the postintervention period in both the vaginal delivery group $(1.4 \%$ to $2.1 \%, p=0.02)$ and the cesarean delivery group $(0.6 \%$ to $1.5 \%, p=0.02)$. 
Table 2: Parameter estimates for interrupted time-series regression models of postpartum length of stay for vaginal and cesarean delivery, January 2012 to December 2016

\begin{tabular}{|c|c|c|c|c|c|c|c|c|}
\hline \multirow[b]{3}{*}{ Parameter } & \multicolumn{4}{|c|}{ Vaginal delivery } & \multicolumn{4}{|c|}{ Cesarean delivery } \\
\hline & \multicolumn{2}{|c|}{ All cases } & \multicolumn{2}{|c|}{ Typical cases* } & \multicolumn{2}{|c|}{ All cases } & \multicolumn{2}{|c|}{ Typical cases* } \\
\hline & Estimate & $\operatorname{Pr}>|t|$ & Estimate & $\operatorname{Pr}>|t|$ & Estimate & $\operatorname{Pr}>|t|$ & Estimate & $\operatorname{Pr}>|t|$ \\
\hline Intercept $\left(\beta_{0}\right)$ & 41.6205 & $<0.0001$ & 43.9919 & $<0.0001$ & 70.9738 & $<0.0001$ & 70.9463 & $<0.0001$ \\
\hline Time $\left(\beta_{1}\right)$ & -0.1088 & 0.0476 & -0.0057 & 0.9077 & 0.0357 & 0.7301 & -0.0132 & 0.8107 \\
\hline Implementation $\left(\beta_{2}\right)$ & -3.6481 & 0.1741 & -5.7028 & 0.0251 & -7.1894 & 0.1664 & -9.4768 & 0.0013 \\
\hline Time after $\left(\beta_{3}\right)$ & -0.0730 & 0.4860 & -0.1750 & 0.0772 & -0.3450 & 0.0941 & -0.2613 & 0.1940 \\
\hline
\end{tabular}

\section{Control analyses}

Analyses of gestational age at admission showed that only the intercept parameters from the models were statistically significant, indicating that the average gestational age at admission remained at about 38-39 weeks throughout the study period (Appendix 1, available at www.cmajopen.ca/content/6/3/ E261/suppl/DC1). In the second control analysis, of hysterectomy performed in a similar patient population, all 3 models (inpatients, same-day admissions and day surgeries) showed that implementation and time after parameters were not statistically significant, indicating that trends in average posthysterectomy length of stay were not associated with implementation of the Monarch Centre (Appendix 1).

\section{Long-term impact of Monarch Centre}

The long-term effect of implementation of the Monarch Centre on postpartum length of stay is shown in Figure 3. These estimates were expressed as absolute and relative differences in observed mean length of stay 1.5 years following implementation and a predicted length of stay assuming constant trend from the preimplantation period (Table 3). The average length of stay following a vaginal birth among all cases was predicted to be 35 hours without the centre, compared to 28.6 hours after the centre was implemented, an absolute reduction of 6.3 hours $(95 \%$ confidence interval 0.9 to 11.8 ), equivalent to an $18 \%$ relative reduction. The absolute reduction increased to 12 hours $(95 \%$ confidence interval 7.2 to 17.2 ) among typical cases. Among all cesarean births, implementation of the centre was associated with an absolute reduction in length of stay of 20 hours (from $73.2 \mathrm{~h}$ to $53.2 \mathrm{~h}$, equivalent to a relative reduction of $27 \%$ ), and this was consistent for the subset of typical cesarean deliveries.

\section{Interpretation}

The salient finding of this study was that the implementation of a community-based postpartum clinic was associated with a significant reduction in postpartum length of stay for vaginal and cesarean deliveries in a large academic teaching hospital.
In addition, there was a decrease in the proportion of women with a length of stay exceeding 48 hours. At 1.5 years following implementation of the Monarch Centre, the average length of stay following a cesarean birth was reduced by 20 hours, or $27 \%$. For vaginal deliveries, length of stay was reduced by 6 hours (18\% relative reduction); among typical cases, the reduction was 12 hours (28\% relative reduction). Among babies, there was an increase in rates of 30-day readmission. No associations were observed in our control analyses between gestational age at delivery or posthysterectomy length of stay and implementation of the centre.

The postpartum period, which extends for at least 6 weeks after childbirth, is known to be associated with important physiologic and psychological changes in the woman. According to the World Health Organization, this intense period is the most critical and yet the most neglected phase in the lives of women and their babies. ${ }^{18}$

Different postpartum management models have been studied and they usually involve home-based, clinical or hospitalbased care. These studies looked at different outcomes including patient satisfaction, breast-feeding rates, cost and hospital readmission for women and neonates. ${ }^{19-21}$ The Monarch Centre was established in January 2014 with the objective of providing support for women during the first 4 weeks after discharge from the hospital. There was institutional support for continuation of the centre given the demonstrated high degree of patient care and satisfaction and no evidence for increases in adverse outcomes. ${ }^{14}$

The results of the present study were largely robust to sensitivity analyses conducted to address the presence of outliers (e.g., a single postpartum length of stay of $950 \mathrm{~h}$ owing to admission to the intensive care unit). Although analysis of all cases and typical cases showed similar findings, it is likely that the Monarch Centre model may be favoured for typical or routine deliveries with fewer complications, and these cases showed greater reductions in length of stay in the current study. The effect of the centre on average length of stay was most robust immediately after the centre was implemented. Although the trends in length of stay indicated further 

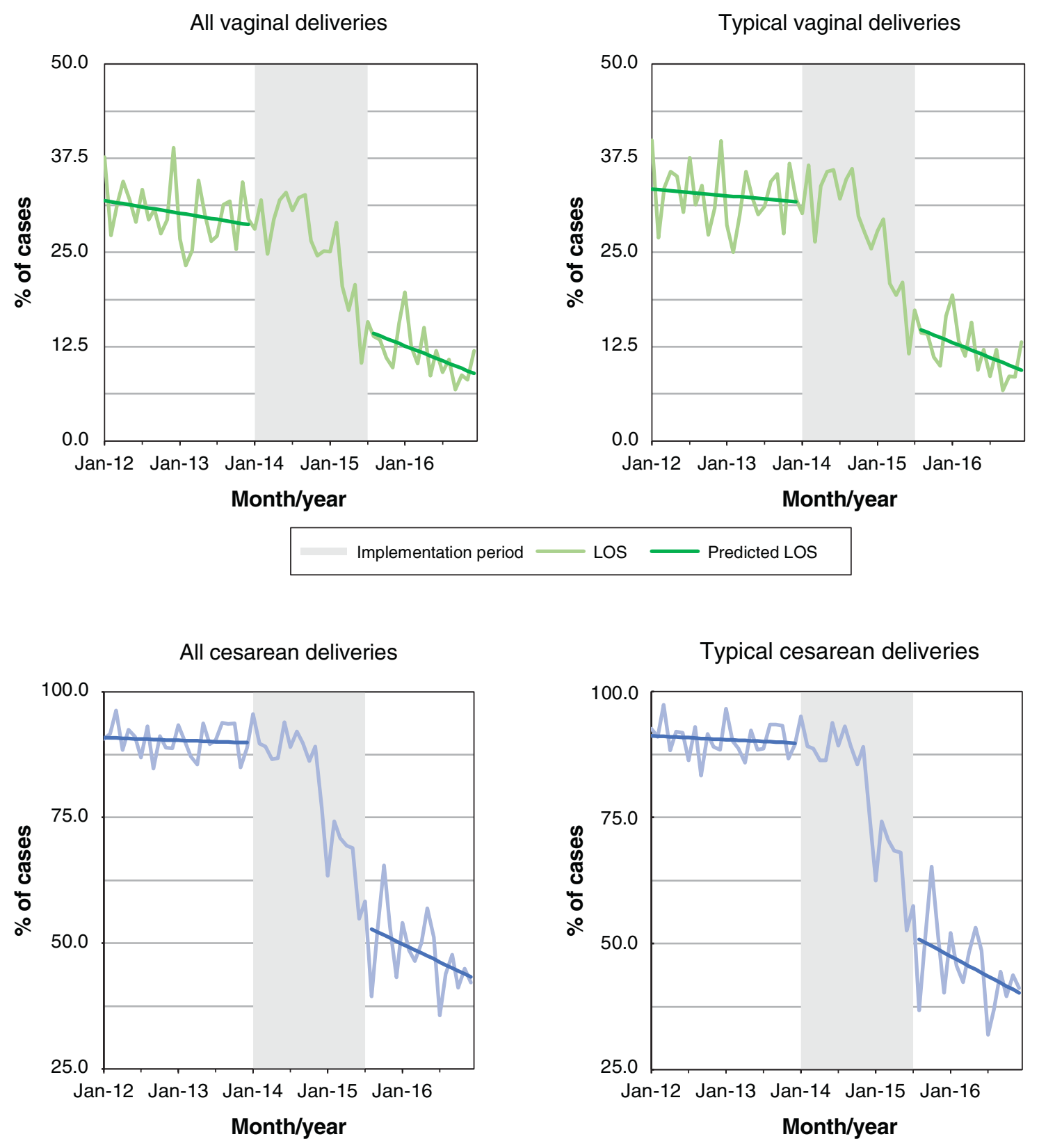

Implementation period

LOS

Predicted LOS

Figure 2: Proportion of women with postpartum length of stay exceeding 48 hours for vaginal and cesarean deliveries, January 2012 to December 2016.

declines in the period after implementation, these effects were less consistent. The rate of 30-day readmission among babies was $2 \%$ overall. This is lower than the rates observed in our previous study (based on self-report) ${ }^{14}$ and in a large study of length of stay and readmission in England. ${ }^{22}$ The significant increase in the 30-day readmission rate over the study period among babies in the present study might be expected as a result of shortening length of stay, and current data do not support lengthening the hospital stay as a method for reducing readmission after vaginal or cesarean birth. ${ }^{22}$ Results of the control analyses add support to our findings that changes in postpartum length of stay were not due to changes in the population, characterized by gestational age at admission, which remained consistent at 38-39 weeks throughout the study period and did not affect other procedures such as hysterectomy. 
Typical vaginal deliveries

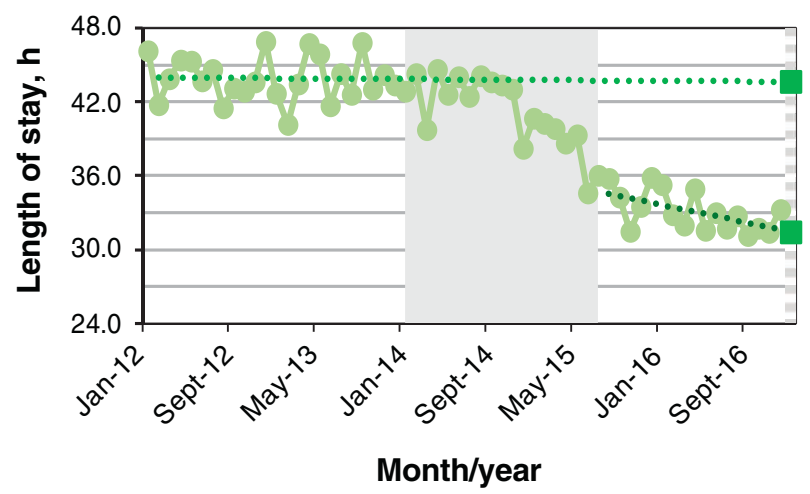

\begin{tabular}{|ll|}
\hline $\begin{array}{l}\text { Implementation period } \\
\text { LOS }\end{array} \ldots$ Forecast period \\
\hline$-\ldots \ldots$ Forecast without clinic \\
\hline
\end{tabular}
Typical cesarean deliveries
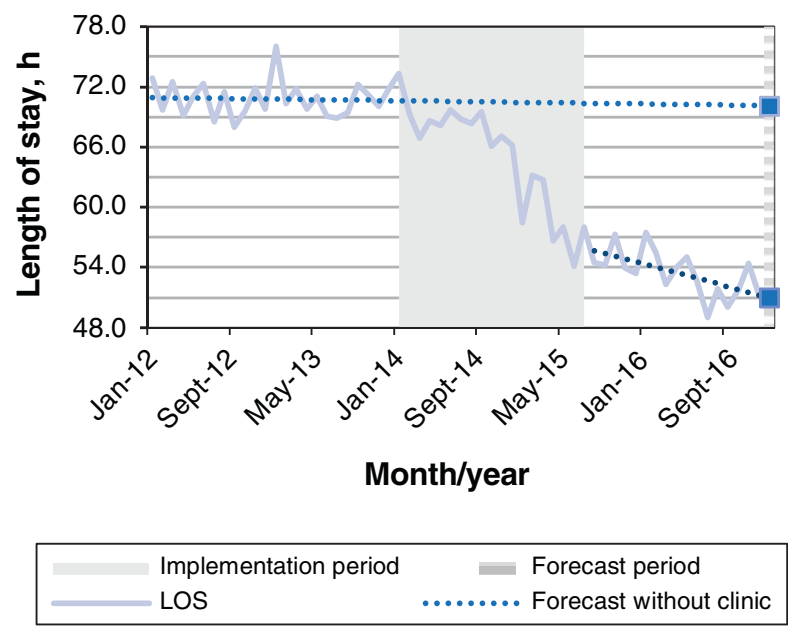

Figure 3: Forecast of average postpartum length of stay (LOS) by month for typical vaginal and cesarean deliveries, January 2012 to December 2016. Darker lines are fitted linear regression lines in the postimplementation period. Squares represent forecasted LOS based on the model.

\begin{tabular}{|c|c|c|}
\hline Variable & All cases & Typical cases only \\
\hline \multicolumn{3}{|l|}{ Vaginal delivery } \\
\hline \multicolumn{3}{|l|}{ Predicted length of stay, $\mathrm{h}$} \\
\hline Without Monarch Centre & 35.0 & 43.6 \\
\hline With Monarch Centre & 28.6 & 31.5 \\
\hline \multicolumn{3}{|l|}{ Difference $(95 \% \mathrm{Cl})$} \\
\hline Absolute & $\begin{array}{c}-6.3 \\
(-11.8 \text { to }-0.9)\end{array}$ & $\begin{array}{c}-12.2 \\
(-17.2 \text { to }-7.2)\end{array}$ \\
\hline Relative & $\begin{array}{c}-0.182 \\
(-0.311 \text { to }-0.052)\end{array}$ & $\begin{array}{c}-0.279 \\
(-0.366 \text { to }-0.192)\end{array}$ \\
\hline \multicolumn{3}{|l|}{ Cesarean delivery } \\
\hline \multicolumn{3}{|l|}{ Predicted length of stay, $h$} \\
\hline Without Monarch Centre & 73.2 & 70.1 \\
\hline With Monarch Centre & 53.2 & 51.0 \\
\hline \multicolumn{3}{|l|}{ Difference $(95 \% \mathrm{Cl})$} \\
\hline Absolute & $\begin{array}{c}-20.0 \\
(-30.4 \text { to }-9.5)\end{array}$ & $\begin{array}{c}19.1 \\
(-24.7 \text { to }-13.6)\end{array}$ \\
\hline Relative & $\begin{array}{c}-0.273 \\
(-0.382 \text { to }-0.164)\end{array}$ & $\begin{array}{c}-0.273 \\
(-0.333 \text { to }-0.212)\end{array}$ \\
\hline
\end{tabular}

\section{Limitations}

This study was conducted at a single clinical centre, and therefore the findings may not be generalizable to other facilities with different patient populations, consultation models or care settings. Similar trends in postpartum length of stay have been seen following the implementation of the Monarch Centre in a sec- ond centre at the General Campus of The Ottawa Hospital. Postpartum length of stay is influenced by multiple others factors related to hospital operations, human resources and the general admitting unit and, in some settings, by maternity stay legislation. ${ }^{23}$ The decision regarding length of the postpartum stay can often be complex, requiring the coordinated effort of different groups including physicians, nurses, ancillary service staff, patients, their families and, in some jurisdictions, insurers. Although level of complexity of the cases was not available within this data set, we tried to address this limitation by stratification by typical cases, which removed cases determined to be more complex owing to transfers, longer hospital stay or complications. There has been a secular trend toward declining average postpartum length of stay in many jurisdictions, alongside reductions in length of stay for all diagnoses, and our observational study within this context therefore may be limited to determine a causal effect of a single program, considering numerous possible confounding factors. We addressed this limitation through a robust analytical approach to separate changes in length of stay during and following implementation of the centre over and above what would have been expected assuming no intervention.

\section{Conclusion}

This study, using a robust design, showed a strong association between the implementation of a community-based multidisciplinary postpartum clinic and decreasing postpartum length of stay beyond what would have been observed owing to secular changes in length of stay. Despite a small increase in rates of 30-day readmission for babies, this alternative model of postpartum care is safe and has the potential for considerable cost savings for providers. Further implementation of alternative models of postpartum care should be considered at the provincial and national levels. 


\section{References}

1. Barua B, Palacios M, Emes J. The sustainability of health care spending in Canada 2017. Vancouver: Fraser Institute; 2017.

2. Inpatient hospitalizations, surgeries, newborns and childbirth indicators, 2015-2016. Ottawa: Canadian Institute for Health Information; 2017

3. Bowers J, Cheyne H. Reducing the length of postnatal hospital stay: implications for cost and quality of care. BMC Health Serv Res 2016;16:16.

4. Mannan I, Rahman SM, Sania A, et al.; Bangladesh Projahnmo Study Group. Can early postpartum home visits by trained community health workers improve breastfeeding of newborns? 7 Perinatol 2008;28:632-40.

5. Paul IM, Beiler JS, Schaefer EW, et al. A randomized trial of single home nursing visits vs office-based care after nursery/maternity discharge: the Nurses for Infants Through Teaching and Assessment After the Nursery (NITTANY) Study. Arch Pediatr Adolesc Med 2012;166:263-70.

6. Maisels MJ, Kring E. Length of stay, jaundice, and hospital readmission. Pediatrics 1998;101:995-8.

7. Salas AA, Salazar J, Burgoa CV, et al. Significant weight loss in breastfed term infants readmitted for hyperbilirubinemia. BMC Pediatr 2009;9:82.

8. Grupp-Phelan J, Taylor JA, Liu LL, et al. Early newborn hospital discharge and readmission for mild and severe jaundice. Arch Pediatr Adolesc Med 1999; 153:1283-8.

9. Catz C, Hanson JW, Simpson L, et al. Summary of workshop: Early discharge and neonatal hyperbilirubinemia. Pediatrics 1995;96:743-5.

10. Britton JR, Britton HL, Beebe SA. Early discharge of the term newborn: a continued dilemma. Pediatrics 1994;94:291-5.

11. De Carolis MP, Cocca C, Valente E, et al. Individualized follow up programme and early discharge in term neonates. Ital f Pediatr 2014;40:70.

12. Escobar GJ, Braveman PA, Ackerson L, et al. A randomized comparison of home visits and hospital-based group follow-up visits after early postpartum discharge. Pediatrics 2001;108:719-27.

13. Dodge KA, Goodman WB, Murphy RA, et al. Implementation and randomized controlled trial evaluation of universal postnatal nurse home visiting. Am 7 Public Health 2014;104(Suppl 1):S136-43.

14. Laliberté C, Dunn S, Pound C, et al. A randomized controlled trial of innovative postpartum care model for mother-baby dyads. PLoS One 2016;11: e0148520.

15. Wong J, Taljaard M, Forster AJ, et al. Derivation and validation of a model to predict daily risk of death in hospital. Med Care 2011;49:734-43.

16. Wagner AK, Soumerai SB, Zhang F, et al. Segmented regression analysis of interrupted time series studies in medication use research. 7 Clin Pharm Ther 2002;27:299-309
17. Grimshaw J, Campbell M, Eccles M, et al. Experimental and quasi-experimental designs for evaluating guideline implementation strategies. Fam Pract 2000;17 (Suppl 1):S11-6.

18. WHO recommendations on postnatal care of the mother and newborn. Geneva: World Health Organization; 2013.

19. Boulvain M, Perneger TV, Othenin-Girard V, et al. Home-based versus hospital-based postnatal care: a randomised trial. BFOG 2004;111:807-13.

20. Gagnon AJ, Edgar L, Kramer MS, et al. A randomized trial of a program of early postpartum discharge with nurse visitation. Am 7 Obstet Gynecol 1997;176:205-11.

21. Lieu TA, Braveman PA, Escobar GJ, et al. A randomized comparison of home and clinic follow-up visits after early postpartum hospital discharge. Pediatrics 2000;105:1058-65.

22. Harron K, Gilbert R, Cromwell D, et al. Newborn length of stay and risk of readmission. Paediatr Perinat Epidemiol 2017;31:221-32.

23. Declercq E, Simmes D. The politics of "Drive-through deliveries": putting early postpartum discharge on the legislative agenda. Milbank Q 1997;75:175-202.

Affiliations: Department of Obstetrics and Gynecology (Hardy), London Hospital, London, Ont.; Quality, Performance and Population Health (Colas, Forster), The Ottawa Hospital; BORN (Better Outcomes Registry and Network) Ontario (Weiss), Children's Hospital of Eastern Ontario and School of Epidemiology and Public Health, University of Ottawa; Monarch Maternal and Newborn Health (Millar); Ottawa Hospital Research Institute, OMNI (Obstetrics \& Maternal Newborn Investigations) Research Group (Walker, Corsi); Department of Obstetrics and Gynecology (Walker), Faculty of Medicine, and School of Epidemiology and Public Health (Corsi), University of Ottawa, Ottawa, Ont.

Contributors: Mark Walker and Daniel Corsi conceived and designed the study. Jo Ann Colas acquired the data and carried out all statistical analyses with input from Deborah Weiss. Ghislain Hardy drafted the manuscript. All of the authors contributed to interpretation of the data and revising the manuscript critically for important intellectual content, gave final approval of the version to be published and agreed to be accountable for all aspects of the work.

Supplemental information: For reviewer comments and the original submission of this manuscript, please see www.cmajopen.ca/content/6/3/ E261/suppl/DC1. 